\title{
Influential Article Review - On Instagram, Endorsers' Digital Marketing Campaigns
}

\author{
Shiloh Wilkes \\ Carmel Chapman
}

\author{
Lyndsey Mccall
}

This paper examines digital marketing. We present insights from a highly influential paper. Here are the highlights from this paper: Social media offers marketers a broad set of instruments to connect to targeted customers in a personalized and interactive manner. Different social media platforms enable different innovative strategies for an organization's social media presence. In this framework, Instagram has become a popular social media platform for luxury fashion brands. This paper explores the area of luxury fashion brands on Instagram by focusing on key practices that should be implemented in a social media marketing strategy. The main objectives of this study are: (1) the present the concept of luxury brands, (2) to examine the social media marketing of luxury brands aimed at driving consumer engagement, (3) to assess the marketing practices of luxury fashion brands on Instagram, especially as they relate to influencers and celebrity endorsements. For our overseas readers, we then present the insights from this paper in Spanish, French, Portuguese, and German.

Keywords: digital marketing, fashion industry, Instagram, luxury brands, social media marketing

\section{SUMMARY}

- This paper addressed multiple objectives, highlighted in the Introduction section, to enhance existing literature in the following areas: the conceptualization of «luxury brands», social media marketing of luxury brands aimed at consumer engagement, specific Instagram marketing practices in the context of luxury fashion brands.

- The basis for this study was the luxury fashion sector where social media strategies have been successfully used to engage and reach customers and aspirational consumers. Academic work dedicated to fashion luxury brands' social media strategy is limited. This paper provides new perspectives on this topic. Previous academic research examined how high-end brands approached Instagram to develop closer relationships with customers and increase the brand awareness in digital settings.

- In this current business environment, social media marketing actions are part of the promotional mix in the new brand communication paradigm. While traditional media have a stronger impact on brand awareness, social media communication strongly influences brand image. Luxury brands have also adopted this platform to reach potential audiences in a creative manner. 
- This study addressed multiple Instagram marketing practices that can be successfully implemented in the context of luxury fashion brands. Through its social media efforts, a company can influence consumers' perceptions, attitudes, and preferences related to their brand, by pursuing practices that aim to develop brand awareness and creating a brand image that resonates with targeted customers. Godey et al. state that «brands should no longer regard social media marketing as a way to reach consumers, but also as an important and cost-effective image-building tool».

- On Instagram, luxury brands need to be proactive in developing original, attention-grabbing content that engages customers. Moreover, original content can also be achieved through partnerships with celebrities and influencers. The created content aims to enhance the brand's legacy and reinforce its uniqueness to potential and current customers, as well as lead to positive behaviors. For instance, in its 2017 report, Instagram reveals that Burberry «led London Fashion Week with $38 \%$ of the discussion thanks to its engaging behind the scenes content».

- Another useful Instagram practice that luxury fashion brands should consider are hashtags. Hashtags represent an effective practice to mobilize the right audiences on Instagram. Moreover, hashtags are used for research and inspiration, by marketers and consumers, alike. Nonetheless, celebrity marketing has a significant role in communicating marketing messages. Similarly, influencer marketing has the advantage of presenting high-end brand in an informal and accessible context. Therefore, luxury brands have to use Instagram in an effective manner to reach targeted audiences.

- One major limitation of this study is the lack of empirical evidence for these propositions.

\section{HIGHLY INFLUENTIAL ARTICLE}

We used the following article as a basis of our evaluation:

Vinerean, S., \& Opreana, A. (2019, December 29). Social Media Marketing Efforts of Luxury Brands on Instagram. Expert Journal of Marketing, 7(2), pp. 144-152.

This is the link to the publisher's website:

https://marketing.expertjournals.com/23446773-714/

\section{INTRODUCTION}

As consumers' engagement rate tends to decrease, luxury brands need to reconsider their digital marketing strategy and discover new ways to maximize their return on marketing spend. A content marketing strategy needs to be adjusted to the appropriate social platform for each targeted audience, while grabbing the attention of key demographics and is, and highlighting compelling calls-to-action with an integrated link to checkout.

Social media platforms represent an essential resource for consumers, whether they use them for leisure activities, interacting with friends, or discovering new products and services. More and more, social media has an important role in the customer journey or decision-making process, influencing every stage from awareness, consideration, evaluation, to purchase and repurchase. For this reason, marketers are highly invested in developing social media strategies aimed at acquiring, retaining, and expanding their customer base. To this end, fashion is considered one of the most important industries since it accounts for a significant part of the worldwide economy (McKinsey\&Co, 2019).

In this industry, luxury fashion plays a key role. A Deloitte report (2017) on millennials' consumer behavior found millennials inclined to purchase luxury say they find information from traditional sources such as magazines, through to videos, websites and blogs. More specifically, 20.5\% of respondents find out about the latest high-end fashion or luxury item trends from social media (Deloitte, 2017). Almost all luxury brands practice social media marketing which is defined as "marketing communications via digital applications, platforms and media that facilitate interaction, collaboration and content sharing among users" 
(Kim and Ko, 2012, p. 1480). Social media offers new opportunities for luxury brands to facilitate the lead qualification process, create brand awareness, and develop the brand as an 'aspirational brand'. According to Tennille Kopiasz, Senior Vice President of Marketing at Parfums Christian Dior, "the best way for luxury brands to inspire consumers has always been through storytelling. As Instagram is a visual storytelling platform, the link is a natural fit." (Instagram, 2017).

In this framework, this study examines this unexplored area of luxury brand marketing on Instagram and examine the key practices high-end brands are currently implementing on social media. The specific objectives of the study are as follows: (1) to examine the concept of luxury brands, (2) to understand the premises social media marketing of luxury brands aimed at driving consumer engagement, (3) to evaluate high-end brands' popular and specific marketing practices on Instagram, particularly related to influencers and celebrity endorsements.

\section{CONCLUSION}

\section{Theoretical Contributions}

This paper addressed multiple objectives, highlighted in the Introduction section, to enhance existing literature in the following areas: (a) the conceptualization of "luxury brands", (b) social media marketing of luxury brands aimed at consumer engagement, (c) specific Instagram marketing practices in the context of luxury fashion brands.

The basis for this study was the luxury fashion sector where social media strategies have been successfully used to engage and reach customers and aspirational consumers. Academic work dedicated to fashion luxury brands' social media strategy is limited. This paper provides new perspectives on this topic. Previous academic research (Godey et al., 2016; Kim and Ko, 2012; Phan et al., 2011) examined how highend brands approached Instagram to develop closer relationships with customers and increase the brand awareness in digital settings.

In this current business environment, social media marketing actions are part of the promotional mix in the new brand communication paradigm (Mangold and Faulds, 2009). While traditional media have a stronger impact on brand awareness, social media communication strongly influences brand image (Godey et al., 2016). Instagram is targeted for luxury brands because of its visual extension that allow brands to have a visual storytelling approach. More and more, Instagram is considered the new destination for inspiration and a new form of 'window shopping' as consumers tend to consult this social media platform especially in the discovery and consideration phase.

Social media represents an essential resource for consumers in their decision-making process, and for marketers in developing and maintaining a close brand-customer relationship. In this framework, Instagram has gained popularity with consumers and marketers, alike. Instagram is a social media platform highly focused on visual storytelling which creates the perfect medium for products and experiences. Luxury brands have also adopted this platform to reach potential audiences in a creative manner (Instagram, 2017).

\section{Managerial Implications}

This study addressed multiple Instagram marketing practices that can be successfully implemented in the context of luxury fashion brands. Through its social media efforts, a company can influence consumers' perceptions, attitudes, and preferences related to their brand, by pursuing practices that aim to develop brand awareness and creating a brand image that resonates with targeted customers. Godey et al. (2016) state that "brands should no longer regard social media marketing as a way to reach consumers, but also as an important and cost-effective image-building tool."

On Instagram, luxury brands need to be proactive in developing original, attention-grabbing content that engages customers. Moreover, original content can also be achieved through partnerships with celebrities and influencers. The created content aims to enhance the brand's legacy and reinforce its uniqueness to potential and current customers, as well as lead to positive behaviors (Herrando et al., 2018). Tools such as boomerangs, highlights, stories, filters, shopping and so on, have proven to be interactive 
practices that give brands multiple creative options to develop the luxury brand image, brand awareness and drive engagement.

Luxury brands who approach Instagram as a social media platform that bridges the gap between the company and targeted consumers need to focus the storytelling. On Instagram, these storytelling capabilities can be achieved through the feed, videos, stories, and live videos. The feed should have an editorial look and be timeless, compared to Stories, where momentary and ephemeral content has a great value. For instance, in its 2017 report, Instagram reveals that Burberry "led London Fashion Week with 38\% of the discussion thanks to its engaging behind the scenes content".

Another useful Instagram practice that luxury fashion brands should consider are hashtags. Hashtags represent an effective practice to mobilize the right audiences on Instagram. Moreover, hashtags are used for research and inspiration, by marketers and consumers, alike. Nonetheless, celebrity marketing has a significant role in communicating marketing messages. Similarly, influencer marketing has the advantage of presenting high-end brand in an informal and accessible context. Therefore, luxury brands have to use Instagram in an effective manner to reach targeted audiences.

\section{Limitations and Future Directions for Expanding the Research}

One major limitation of this study is the lack of empirical evidence for these propositions. Future research should focus on developing a quantitative marketing research with primary data to examine the level of Instagram use to influence luxury fashion purchases, in general and related to a particular high-end brand. Another idea for expanding this study is to conduct a qualitative research based on text mining and sentiment analysis to gather new information from users' comments about their use and perception of luxury fashion brands' presence on Instagram. The study could also be extended in developing a cross-cultural research that examines consumers' use of Instagram and how they perceive social media marketing practices of luxury fashion brands.

\section{REFERENCES}

Amaldoss, W., \& Jain, S. (2005). Conspicuous consumption and sophisticated thinking. Management Science, 51(10), 1449-1466.

Berthon, P., Pitt, L., Parent, M., \& Berthon, J-P. (2009). Aesthetics and ephemerality: Observing and preserving the luxury brand. California Management Review, 52(1), 45-66.

Beverland, M.B. (2004). Uncovering 'Theories-in-Use': Building Luxury Wine Brands. European Journal of Marketing, 38(3/4), 446-466.

Brodie, R.J., Hollebeek, L.D., Juric, B., \& Ilic, A. (2011). Customer engagement: Conceptual domain, fundamental propositions, and implications for research. Journal of Service Research, 14(3), 252-271. doi: 10.1177/1094670511411703

Brodie, R.J., Ilic, A., Juric, B., \& Hollebeek, L. (2013). Consumer engagement in a virtual brand community: An exploratory analysis. Journal of Business Research, 66(1), 105-114.

Casaló, L.V., Flavián, C., \& Ibáñez-Sánchez, S. (2018). Influencers on Instagram: Antecedents and consequences of opinion leadership. Journal of Business Research. doi:10.1016/j.jbusres.2018.07.005

Chu, S.C., Kamal, S., \& Kim, Y. (2013). Understanding consumers' responses toward social media advertising and purchase intention toward luxury products. Journal of Global Fashion Marketing, $4(3), 158-174$.

Chu, S.C., Kamal, S., \& Kim, Y. (2019). Re-examining of consumers' responses toward social media advertising and purchase intention toward luxury products from 2013 to 2018: A retrospective commentary. Journal of Global Fashion Marketing, 10(1), 81-92. doi:10.1080/20932685.2018.1550008

De Veirman, M., Cauberghe, V., \& Hudders, L. (2017). Marketing through Instagram influencers: The impact of number of followers and product divergence on brand attitude. International Journal of Advertising, 36(5), 798-828. doi: 10.1080/02650487.2017.1348035 
Del Rocío Bonilla, M., del Olmo Arriaga, J.L., \& Andreu, D. (2019). The interaction of Instagram followers in the fast fashion sector: The case of Hennes and Mauritz (H\&M). Journal of Global Fashion Marketing, 10(4), 342-357. doi: 10.1080/20932685.2019.1649168

Deloitte. (2017). Bling it on What makes a millennial spend more? Retrieved October 2019, from https://www2.deloitte.com/content/dam/Deloitte/uk/Documents/consumer-business/deloitte-ukyoung-luxury-shopper-2017.pdf?utm_source=luxe.digital\&utm_campaign=luxe-digital-luxurynews

Dhaoui, C. (2014). An Empirical Study of Luxury Brand Marketing Effectiveness and Its Impact on Consumer Engagement on Facebook. Journal of Global Fashion Marketing, 5(3), 209-222.

Djafarova, E., \& Rushworth, C. (2017). Exploring the credibility of online celebrities' Instagram profiles in influencing the purchase decisions of young female users. Computers in Human Behavior, 68, 1-7. doi:10.1016/j.chb.2016.11.009

Fionda, A.M., \& Moore, C.M. (2009). The anatomy of the luxury fashion brand. Journal of Brand Management, 16(5-6), 347-363. doi:10.1057/bm.2008.45

Gao, W., Li, Q., \& Drougas, A. (2017). Ownership Structure and Stock Price Crash Risk: Evidence from China. Journal of Applied Business and Economics, 19(4). Retrieved from https://articlegateway.com/index.php/JABE/article/view/715

Glassman, M. (2019). The World's Biggest Influencers Helped Lift Luxury Brands on Instagram [online]. Retrieved October 20, 2019, from https://www.bloomberg.com/news/features/2019-10-22/theseinfluencers-drove-the-most-engagement-for-instagram-brands

Globalwebindex. (2015). Profiling Instagram's future on its fifth birthday. Retrieved October 20, 2019, from https://blog.globalwebindex.com/trends/profiling-instagram-s-future-on-its-fifth-birthday/

Godey, B., Manthiou, A., Pederzoli, D., Rokka, J., Aiello, G., Donvito, R., \& Singh, R. (2016). Social media marketing efforts of luxury brands: Influence on brand equity and consumer behavior. Journal of Business Research, 69(12), 5833-5841. doi:10.1016/j.jbusres.2016.04.181

\#Hashoff. (2017). Influencer marketer. A \#Hashoff state of the union report. Retrieved October 2019, from https://www.hashoff.com/

Herrando, C., Jimenez-Martinez, J., \& Martin De Hoyos, M.J. (2018). Surfing or flowing? How to retain e-customers on the internet. Spanish Journal of Marketing-ESIC, 22(1), 2-21.

Hollebeek, L., Glynn, M.S., \& Brodie, R.J. (2014). Consumer brand engagement in social media: Conceptualization, scale development and validation. Journal of Interactive Marketing, 28(2), $149-165$.

Instagram. (2017). Experience Luxury on Instagram. Retrieved October 20, 2019, from https://business.instagram.com/a/insights/luxury

Instagram. (2019). Instagram Shopping. Retrieved October 20, 2019, from https://www.facebook.com/business/instagram/shopping

Jackson, T.B. (2004). International Retail Marketing. Oxford: Elsevier Butterworth-Heinemann.

Kim, A.J., \& Ko, E. (2010). Impacts of Luxury Fashion Brand's Social Media Marketing on Customer Relationship and Purchase Intention. Journal of Global Fashion Marketing, 1(3), 164-171. doi:10.1080/20932685.2010.10593068

Kim, A.J., \& Ko, E. (2012). Do Social Media Marketing Activities Enhance Customer Equity? An Empirical Study of Luxury Fashion Brand. Journal of Business Research, 65(10), 1480-1486.

Ko, E., Costello, J.P., \& Taylor, C.R. (2017). What is a luxury brand? A new definition and review of the literature. Journal of Business Research, 99, 405-413.

Lee, J.E., \& Watkins, B. (2016). YouTube vloggers' influence on consumer luxury brand perceptions and intentions. Journal of Business Research, 69(12), 5753-5760. doi:10.1016/j.jbusres.2016.04.171

Locowise. (2017). Instagram follower growth is now lagging behind Facebook page likes growth. Retrieved September 11, 2019, from https://locowise.com/blog/instagram-follower-growth-isnow-lagging-behind-facebook-page-likes-growth

Mangold, W.G., \& Faulds, D.J. (2009). Social media: The new hybrid element of the promotion mix. Business Horizons, 52, 357-365. 
McCormick, K. (2016). Celebrity endorsements: Influence of a product-endorser match on Millennials attitudes and purchase intentions. Journal of Retailing and Consumer Services, 32, 39-45.

McKinsey\&Company. (2019). The State of Fashion 2020: Navigating uncertainty. Business of Fashion and McKinsey\&Company. Retrieved from

https://www.mckinsey.com/ /media/McKinsey/Industries/Retail/Our\%20Insights/The\%20state\% $20 \mathrm{of} \% 20$ fashion $\% 202020 \% 20$ Navigating\%20uncertainty/The-State-of-Fashion-2020-final.ashx

Okonkwo, U. (2009). Sustaining the Luxury Brand on the Internet. Journal of Brand Management, 16 $(5 / 6), 302-310$.

Pentina, I., Guilloux, V., \& Micu, A.C. (2018). Exploring Social Media Engagement Behaviors in the Context of Luxury Brands. Journal of Advertising, 47(1), 55-69. doi: 10.1080/00913367.2017.1405756

Phan, M., Thomas, R., \& Heine, K. (2011). Social Media and Luxury Brand Management: The Case of Burberry. Journal of Global Fashion Marketing, 2(4), 213-222. doi:10.1080/20932685.2011.10593099

Pradhan, D., Duraipandian, I., \& Sethi, D. (2014). Celebrity endorsement: How celebrity- brand-user personality congruence affects brand attitude and purchase intention. Journal of Marketing Communications, 22(5), 456-473.

Schiffman, L.G., Hansen, H., \& Kanuk, L. (2012). Consumer behaviour. London: Prentice Hall.

Statista.com. (2019). Leading luxury brands with the most followers on Instagram as of May 2019. Retrieved from https://www.statista.com/statistics/483753/leading-luxury-brands-instagramfollowers/

Tynan, C., McKechnie, S., \& Chhuon, C. (2010). Co-Creating Value for Luxury Brands. Journal of Business Research, 63(11), 1156-1163.

Watts, D.J., \& Dodds, P.S. (2007). Influentials, networks, and public opinion formation. Journal of Consumer Research, 34(4), 441-58. doi:10.1086/518527

Wiedman, K.P., Hennings, N., \& Langner, S. (2010). Spreading the word of fashion: Identifying social influencers in fashion marketing. Journal of Global Fashion Marketing, 1(3), 142-153.

\section{APPENDIX}

\section{FIGURE 1 \\ NO. OF INSTAGRAM FOLLOWERS (IN MILLIONS) FOR LUXURY FASHION BRANDS AS OF MAY 2019}

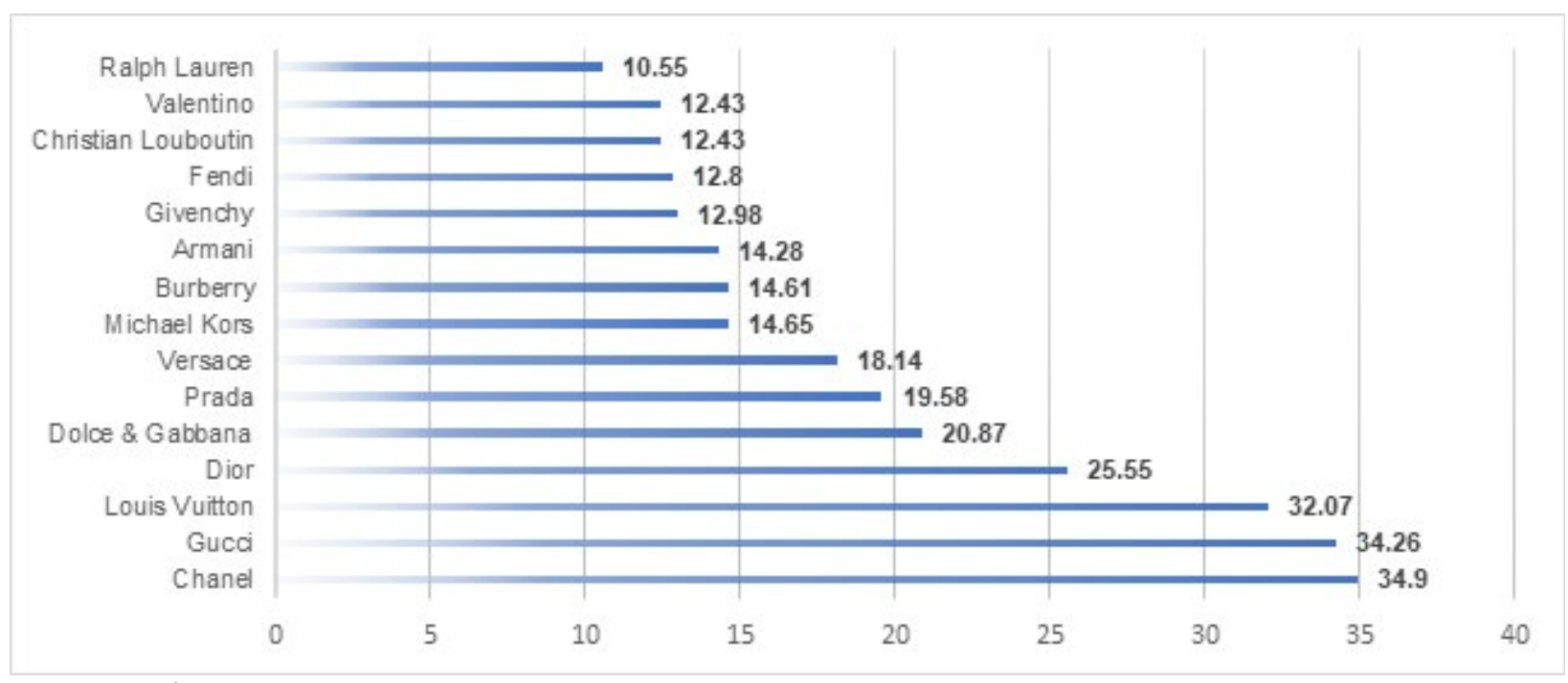

Source: Statista.com, 2019 
FIGURE 2

THE USE OF INSTAGRAM SHOPPING BY LUXURY BRAND “TOM FORD”(@tomford)

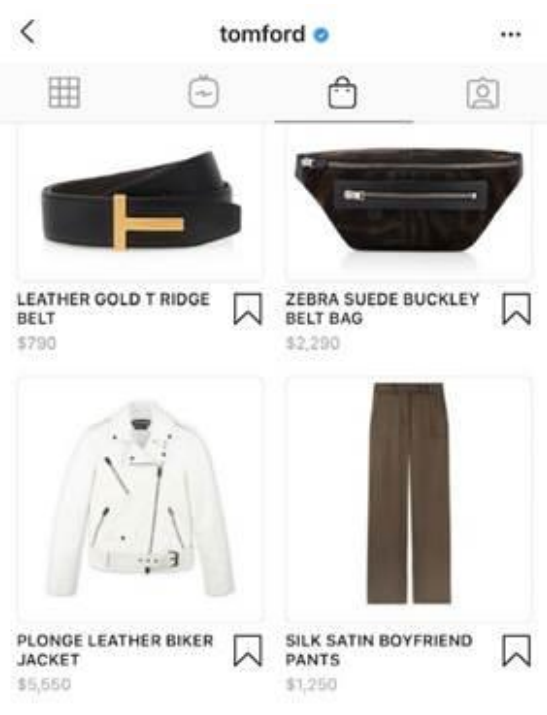

FIGURE 3

THE USE OF INSTAGRAM HIGHLIGHTS AND IGTV BY “VALENTINO”(@maisonvalentino)

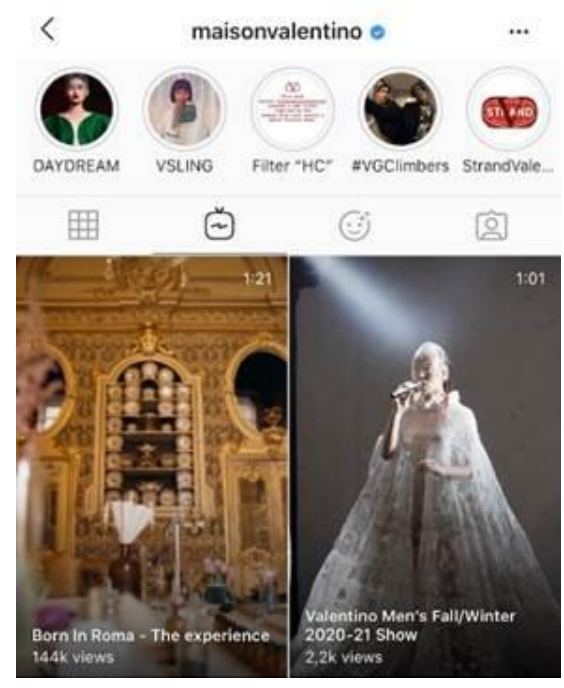




\section{FIGURE 4}

\section{THE USE OF INSTAGRAM HIGHLIGHTS AND AR FILTERS BY "BALMAIN"(@balmain)}

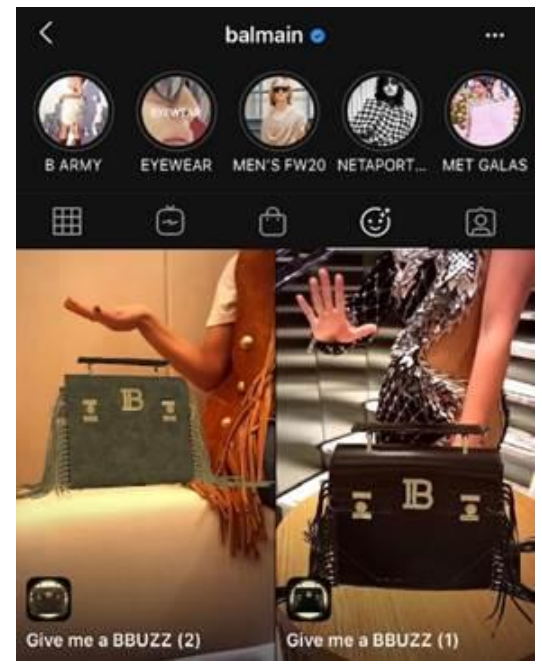

\section{TRANSLATED VERSION: SPANISH}

Below is a rough translation of the insights presented above. This was done to give a general understanding of the ideas presented in the paper. Please excuse any grammatical mistakes and do not hold the original authors responsible for these mistakes.

\section{VERSION TRADUCIDA: ESPAÑOL}

A continuación se muestra una traducción aproximada de las ideas presentadas anteriormente. Esto se hizo para dar una comprensión general de las ideas presentadas en el documento. Por favor, disculpe cualquier error gramatical y no responsabilite a los autores originales de estos errores.

\section{INTRODUCCIÓN}

A medida que la tasa de participación de los consumidores tiende a disminuir, las marcas de lujo deben reconsiderar su estrategia de marketing digital y descubrir nuevas formas de maximizar su retorno del gasto en marketing. Una estrategia de marketing de contenido debe ajustarse a la plataforma social adecuada para cada público objetivo, al tiempo que capta la atención de los grupos demográficos clave y sus necesidades, y destaca las llamadas a la acción convincentes con un enlace integrado al pago.

Las plataformas de redes sociales representan un recurso esencial para los consumidores, ya sea que las utilicen para actividades de ocio, interactuar con amigos o descubrir nuevos productos y servicios. Cada vez más, las redes sociales tienen un papel importante en el viaje del cliente o el proceso de toma de decisiones, influyendo en cada etapa, desde la concienciación, la consideración, la evaluación hasta la compra y la recompra. Por esta razón, los especialistas en marketing invierten mucho en el desarrollo de estrategias de redes sociales destinadas a adquirir, retener y expandir su base de clientes. Para ello, la moda es considerada una de las industrias más importantes ya que representa una parte significativa de la economía mundial (McKinsey\&Co, 2019).

En esta industria, la moda de lujo juega un papel clave. Un informe de Deloitte (2017) sobre el comportamiento del consumidor de los millennials encontró que los millennials inclinados a comprar lujo dicen que encuentran información de fuentes tradicionales como revistas, hasta videos, sitios web y blogs. Más específicamente, el $20.5 \%$ de los encuestados se entera de las últimas tendencias de moda o artículos de lujo de alta gama de las redes sociales (Deloitte, 2017). Casi todas las marcas de lujo practican el 
marketing en redes sociales, que se define como "comunicaciones de marketing a través de aplicaciones digitales, plataformas y medios que facilitan la interacción, la colaboración y el intercambio de contenido entre los usuarios" (Kim y Ko, 2012, p. 1480). Las redes sociales ofrecen nuevas oportunidades para que las marcas de lujo faciliten el proceso de calificación de clientes potenciales, creen conciencia de marca y desarrollen la marca como una "marca aspiracional". Según Tennille Kopiasz, vicepresidente senior de marketing de Parfums Christian Dior, "la mejor manera para que las marcas de lujo inspiren a los consumidores siempre ha sido a través de la narración de historias. Como Instagram es una plataforma de narración visual, el enlace es un ajuste natural". (Instagram, 2017).

En este marco, este estudio examina esta área inexplorada del marketing de marcas de lujo en Instagram y examina las prácticas clave que las marcas de alta gama están implementando actualmente en las redes sociales. Los objetivos específicos del estudio son los siguientes: (1) examinar el concepto de marcas de lujo, (2) comprender las premisas del marketing en redes sociales de las marcas de lujo destinadas a impulsar el compromiso del consumidor, (3) evaluar las prácticas de marketing populares y específicas de las marcas de alta gama en Instagram, particularmente relacionadas con los influencers y los respaldos de celebridades.

\section{CONCLUSIÓN}

\section{Contribuciones Teóricas}

Este documento abordó múltiples objetivos, destacados en la sección de Introducción, para mejorar la literatura existente en las siguientes áreas: (a) la conceptualización de las "marcas de lujo", (b) el marketing en redes sociales de marcas de lujo dirigido a la participación del consumidor, (c) prácticas específicas de marketing de Instagram en el contexto de las marcas de moda de lujo.

La base de este estudio fue el sector de la moda de lujo, donde las estrategias de redes sociales se han utilizado con éxito para atraer y llegar a clientes y consumidores aspiracionales. El trabajo académico dedicado a la estrategia de redes sociales de las marcas de moda de lujo es limitado. Este documento proporciona nuevas perspectivas sobre este tema. Investigación académica previa (Godey et al., 2016; Kim y Ko, 2012; Phan et al., 2011) examinaron cómo las marcas de alta gama se acercaron a Instagram para desarrollar relaciones más cercanas con los clientes y aumentar el conocimiento de la marca en entornos digitales.

En este entorno empresarial actual, las acciones de marketing en redes sociales forman parte del mix promocional en el nuevo paradigma de comunicación de marca (Mangold y Faulds, 2009). Mientras que los medios tradicionales tienen un mayor impacto en el conocimiento de la marca, la comunicación en las redes sociales influye fuertemente en la imagen de la marca (Godey et al., 2016). Instagram está dirigido a marcas de lujo debido a su extensión visual que permite a las marcas tener un enfoque de narración visual. Cada vez más, Instagram se considera el nuevo destino para la inspiración y una nueva forma de 'escaparate', ya que los consumidores tienden a consultar esta plataforma de redes sociales, especialmente en la fase de descubrimiento y consideración.

Las redes sociales representan un recurso esencial para los consumidores en su proceso de toma de decisiones, y para los especialistas en marketing en el desarrollo y mantenimiento de una estrecha relación marca-cliente. En este marco, Instagram ha ganado popularidad entre los consumidores y los especialistas en marketing, por igual. Instagram es una plataforma de redes sociales altamente enfocada en la narración visual que crea el medio perfecto para productos y experiencias. Las marcas de lujo también han adoptado esta plataforma para llegar a audiencias potenciales de una manera creativa (Instagram, 2017).

\section{Implicaciones Gerenciales}

Este estudio abordó múltiples prácticas de marketing de Instagram que se pueden implementar con éxito en el contexto de las marcas de moda de lujo. A través de sus esfuerzos en las redes sociales, una empresa puede influir en las percepciones, actitudes y preferencias de los consumidores relacionadas con su marca, mediante la aplicación de prácticas que tienen como objetivo desarrollar el conocimiento de la marca y crear una imagen de marca que resuene con los clientes objetivo. Godey et al. (2016) afirman que 
"las marcas ya no deben considerar el marketing en redes sociales como una forma de llegar a los consumidores, sino también como una herramienta de construcción de imágenes importante y rentable".

En Instagram, las marcas de lujo deben ser proactivas en el desarrollo de contenido original y llamativo que atraiga a los clientes. Además, el contenido original también se puede lograr a través de asociaciones con celebridades e influencers. El contenido creado tiene como objetivo mejorar el legado de la marca y reforzar su singularidad para los clientes potenciales y actuales, así como conducir a comportamientos positivos (Herrando et al., 2018). Herramientas como boomerangs, highlights, historias, filtros, compras, etc., han demostrado ser prácticas interactivas que brindan a las marcas múltiples opciones creativas para desarrollar la imagen de marca de lujo, el conocimiento de la marca e impulsar el compromiso.

Las marcas de lujo que se acercan a Instagram como una plataforma de redes sociales que cierra la brecha entre la empresa y los consumidores objetivo deben centrarse en la narración de historias. En Instagram, estas capacidades de narración de historias se pueden lograr a través del feed, videos, historias y videos en vivo. El feed debe tener un aspecto editorial y ser atemporal, en comparación con stories, donde el contenido momentáneo y efímero tiene un gran valor. Por ejemplo, en su informe de 2017, Instagram revela que Burberry "lideró la Semana de la Moda de Londres con el 38\% de la discusión gracias a su atractivo contenido detrás de escena".

Otra práctica útil de Instagram que las marcas de moda de lujo deben considerar son los hashtags. Los hashtags representan una práctica efectiva para movilizar a las audiencias adecuadas en Instagram. Además, los hashtags se utilizan para la investigación y la inspiración, tanto por los especialistas en marketing como por los consumidores. Sin embargo, el marketing de celebridades tiene un papel importante en la comunicación de mensajes de marketing. Del mismo modo, el marketing de influencers tiene la ventaja de presentar la marca de alta gama en un contexto informal y accesible. Por lo tanto, las marcas de lujo tienen que usar Instagram de manera efectiva para llegar a audiencias específicas.

\section{Limitaciones Y Direcciones Futuras Para Expandir La Investigación}

Una limitación importante de este estudio es la falta de evidencia empírica para estas proposiciones. La investigación futura debe centrarse en el desarrollo de una investigación de marketing cuantitativa con datos primarios para examinar el nivel de uso de Instagram para influir en las compras de moda de lujo, en general y relacionadas con una marca de alta gama en particular. Otra idea para ampliar este estudio es realizar una investigación cualitativa basada en la minería de texto y el análisis de sentimientos para recopilar nueva información de los comentarios de los usuarios sobre su uso y percepción de la presencia de las marcas de moda de lujo en Instagram. El estudio también podría ampliarse en el desarrollo de una investigación intercultural que examine el uso de Instagram por parte de los consumidores y cómo perciben las prácticas de marketing en redes sociales de las marcas de moda de lujo.

\section{TRANSLATED VERSION: FRENCH}

Below is a rough translation of the insights presented above. This was done to give a general understanding of the ideas presented in the paper. Please excuse any grammatical mistakes and do not hold the original authors responsible for these mistakes.

\section{VERSION TRADUITE: FRANÇAIS}

Voici une traduction approximative des idées présentées ci-dessus. Cela a été fait pour donner une compréhension générale des idées présentées dans le document. Veuillez excuser toutes les erreurs grammaticales et ne pas tenir les auteurs originaux responsables de ces erreurs.

\section{INTRODUCTION}

Alors que le taux d'engagement des consommateurs a tendance à diminuer, les marques de luxe doivent reconsidérer leur stratégie de marketing numérique et découvrir de nouvelles façons de maximiser leur 
retour sur les dépenses marketing. Une stratégie de marketing de contenu doit être adaptée à la plate-forme sociale appropriée pour chaque public ciblé, tout en attirant l'attention des données démographiques clés et en mettant en évidence des appels à l'action convaincants avec un lien intégré vers le paiement.

Les plateformes de médias sociaux représentent une ressource essentielle pour les consommateurs, qu'ils les utilisent pour des activités de loisirs, interagir avec des amis ou découvrir de nouveaux produits et services. De plus en plus, les médias sociaux jouent un rôle important dans le parcours client ou le processus de prise de décision, influençant chaque étape, de la sensibilisation, de la considération, de l'évaluation, à l'achat et au rachat. Pour cette raison, les spécialistes du marketing sont fortement investis dans le développement de stratégies de médias sociaux visant à acquérir, fidéliser et élargir leur clientèle. À cette fin, la mode est considérée comme l'une des industries les plus importantes puisqu'elle représente une part importante de l'économie mondiale (McKinsey \& Co, 2019).

Dans cette industrie, la mode de luxe joue un rôle clé. Un rapport de Deloitte (2017) sur le comportement des consommateurs de la génération $Y$ a révélé que les milléniaux enclins à acheter du luxe disent qu'ils trouvent des informations provenant de sources traditionnelles telles que des magazines, des vidéos, des sites Web et des blogs. Plus précisément, 20,5\% des répondants découvrent les dernières tendances en matière de mode ou d'articles de luxe haut de gamme à partir des médias sociaux (Deloitte, 2017). Presque toutes les marques de luxe pratiquent le marketing des médias sociaux qui est défini comme «des communications marketing via des applications numériques, des plateformes et des médias qui facilitent l'interaction, la collaboration et le partage de contenu entre les utilisateurs » (Kim et Ko, 2012, p. 1480). Les médias sociaux offrent de nouvelles opportunités aux marques de luxe pour faciliter le processus de qualification des prospects, créer une notoriété de la marque et développer la marque en tant que « marque ambitieuse ». Selon Tennille Kopiasz, Senior Vice President marketing chez Parfums Christian Dior, « la meilleure façon pour les marques de luxe d'inspirer les consommateurs a toujours été de raconter des histoires. Comme Instagram est une plate-forme de narration visuelle, le lien est un ajustement naturel. (Instagram, 2017).

Dans ce cadre, cette étude examine ce domaine inexploré du marketing des marques de luxe sur Instagram et examine les pratiques clés que les marques haut de gamme mettent actuellement en œuvre sur les médias sociaux. Les objectifs spécifiques de l'étude sont les suivants: (1) examiner le concept de marques de luxe, (2) comprendre les prémisses du marketing sur les médias sociaux des marques de luxe visant à stimuler l'engagement des consommateurs, (3) évaluer les pratiques marketing populaires et spécifiques des marques haut de gamme sur Instagram, en particulier en ce qui concerne les influenceurs et les approbations de célébrités.

\section{CONCLUSION}

\section{Contributions Théoriques}

Ce document abordait de multiples objectifs, mis en évidence dans la section Introduction, pour améliorer la littérature existante dans les domaines suivants: (a) la conceptualisation des «marques de luxe », (b) le marketing des médias sociaux des marques de luxe visant à l'engagement des consommateurs, (c) les pratiques de marketing Instagram spécifiques dans le contexte des marques de mode de luxe.

La base de cette étude était le secteur de la mode de luxe où les stratégies de médias sociaux ont été utilisées avec succès pour engager et atteindre les clients et les consommateurs ambitieux. Les travaux académiques consacrés à la stratégie des marques de luxe de mode sur les médias sociaux sont limités. Cet article offre de nouvelles perspectives sur ce sujet. Recherches universitaires antérieures (Godey et coll., 2016; Kim et Ko, 2012; Phan et al., 2011) ont examiné comment les marques haut de gamme ont approché Instagram pour développer des relations plus étroites avec les clients et augmenter la notoriété de la marque dans les environnements numériques.

Dans l'environnement commercial actuel, les actions de marketing sur les médias sociaux font partie du mix promotionnel dans le nouveau paradigme de communication de marque (Mangold et Faulds, 2009). Alors que les médias traditionnels ont un impact plus fort sur la notoriété de la marque, la communication sur les médias sociaux influence fortement l'image de marque (Godey et al., 2016). Instagram est ciblé pour 
les marques de luxe en raison de son extension visuelle qui permet aux marques d'avoir une approche de narration visuelle. De plus en plus, Instagram est considéré comme la nouvelle destination d'inspiration et une nouvelle forme de "lèche-vitrine », car les consommateurs ont tendance à consulter cette plate-forme de médias sociaux, en particulier dans la phase de découverte et de considération.

Les médias sociaux représentent une ressource essentielle pour les consommateurs dans leur processus de prise de décision et pour les spécialistes du marketing dans le développement et le maintien d'une relation marque-client étroite. Dans ce cadre, Instagram a gagné en popularité auprès des consommateurs et des spécialistes du marketing. Instagram est une plate-forme de médias sociaux fortement axée sur la narration visuelle qui crée le support idéal pour les produits et les expériences. Les marques de luxe ont également adopté cette plateforme pour atteindre des publics potentiels de manière créative (Instagram, 2017).

\section{Implications Managériales}

Cette étude a abordé de multiples pratiques de marketing Instagram qui peuvent être mises en œuvre avec succès dans le contexte des marques de mode de luxe. Grâce à ses efforts sur les médias sociaux, une entreprise peut influencer les perceptions, les attitudes et les préférences des consommateurs liées à leur marque, en poursuivant des pratiques visant à développer la notoriété de la marque et en créant une image de marque qui résonne avec les clients ciblés. Godey et al. (2016) affirment que « les marques ne devraient plus considérer le marketing des médias sociaux comme un moyen d'atteindre les consommateurs, mais aussi comme un outil important et rentable de création d'image ».

Sur Instagram, les marques de luxe doivent être proactives dans le développement de contenu original et captivant qui engage les clients. De plus, le contenu original peut également être réalisé grâce à des partenariats avec des célébrités et des influenceurs. Le contenu créé vise à améliorer l'héritage de la marque et à renforcer son caractère unique auprès des clients potentiels et actuels, ainsi qu'à conduire à des comportements positifs (Herrando et al., 2018). Des outils tels que les boomerangs, les faits saillants, les histoires, les filtres, le shopping, etc., se sont avérés être des pratiques interactives qui offrent aux marques de multiples options créatives pour développer l'image de marque de luxe, la notoriété de la marque et stimuler l'engagement.

Les marques de luxe qui abordent Instagram comme une plate-forme de médias sociaux qui comble le fossé entre l'entreprise et les consommateurs ciblés doivent se concentrer sur la narration. Sur Instagram, ces capacités de narration peuvent être obtenues via le flux, les vidéos, les histoires et les vidéos en direct. Le flux doit avoir un aspect éditorial et être intemporel, par rapport aux Stories, où le contenu momentané et éphémère a une grande valeur. Par exemple, dans son rapport de 2017, Instagram révèle que Burberry « a mené la Fashion Week de Londres avec 38\% de la discussion grâce à son contenu engageant dans les coulisses $»$.

Une autre pratique Instagram utile que les marques de mode de luxe devraient considérer sont les hashtags. Les hashtags représentent une pratique efficace pour mobiliser les bons publics sur Instagram. De plus, les hashtags sont utilisés pour la recherche et l'inspiration, par les spécialistes du marketing et les consommateurs. Néanmoins, le marketing des célébrités joue un rôle important dans la communication des messages marketing. De même, le marketing d'influence a l'avantage de présenter une marque haut de gamme dans un contexte informel et accessible. Par conséquent, les marques de luxe doivent utiliser Instagram de manière efficace pour atteindre des publics ciblés.

\section{Limites Et Orientations Futures Pour L'expansion De La Recherche}

L'une des principales limites de cette étude est le manque de preuves empiriques de ces propositions. Les recherches futures devraient se concentrer sur le développement d'une étude marketing quantitative avec des données primaires pour examiner le niveau d'utilisation d'Instagram pour influencer les achats de mode de luxe, en général et liés à une marque haut de gamme particulière. Une autre idée pour élargir cette étude est de mener une recherche qualitative basée sur l'exploration de texte et l'analyse des sentiments pour recueillir de nouvelles informations à partir des commentaires des utilisateurs sur leur utilisation et leur perception de la présence des marques de mode de luxe sur Instagram. L'étude pourrait également être 
étendue au développement d'une recherche interculturelle qui examine l'utilisation d'Instagram par les consommateurs et la façon dont ils perçoivent les pratiques de marketing des marques de mode de luxe sur les médias sociaux.

\section{TRANSLATED VERSION: GERMAN}

Below is a rough translation of the insights presented above. This was done to give a general understanding of the ideas presented in the paper. Please excuse any grammatical mistakes and do not hold the original authors responsible for these mistakes.

\section{ÜBERSETZTE VERSION: DEUTSCH}

Hier ist eine ungefähre Übersetzung der oben vorgestellten Ideen. Dies wurde getan, um ein allgemeines Verständnis der in dem Dokument vorgestellten Ideen zu vermitteln. Bitte entschuldigen Sie alle grammatikalischen Fehler und machen Sie die ursprünglichen Autoren nicht für diese Fehler verantwortlich.

\section{EINLEITUNG}

Da die Engagement-Rate der Verbraucher tendenziell abnimmt, müssen Luxusmarken ihre digitale Marketingstrategie überdenken und neue Wege finden, um ihre Rendite auf Marketingausgaben zu maximieren. Eine Content-Marketing-Strategie muss an die geeignete soziale Plattform für jede Zielgruppe angepasst werden, wobei die Aufmerksamkeit der wichtigsten demografischen Merkmale und Is auf sich gezogen und überzeugende Handlungsaufforderungen mit einem integrierten Link zur Kasse hervorgehoben werden müssen.

Social-Media-Plattformen stellen eine wesentliche Ressource für Verbraucher dar, unabhängig davon, ob sie sie für Freizeitaktivitäten, die Interaktion mit Freunden oder die Entdeckung neuer Produkte und Dienstleistungen nutzen. Social Media spielt immer mehr eine wichtige Rolle in der Customer Journey oder im Entscheidungsprozess und beeinflusst jede Phase von der Bekanntheit, Überlegung, Bewertung bis hin zum Kauf und Rückkauf. Aus diesem Grund sind Vermarkter stark in die Entwicklung von Social-MediaStrategien investiert, die darauf abzielen, ihren Kundenstamm zu gewinnen, zu halten und zu erweitern. $\mathrm{Zu}$ diesem Zweck gilt die Mode als eine der wichtigsten Branchen, da sie einen bedeutenden Teil der Weltwirtschaft ausmacht (McKinsey\&Co, 2019).

In dieser Branche spielt Luxusmode eine Schlüsselrolle. Ein Deloitte-Bericht (2017) über das Verbraucherverhalten von Millennials ergab, dass Millennials, die dazu neigen, Luxus zu kaufen, angeben, dass sie Informationen aus traditionellen Quellen wie Zeitschriften bis hin zu Videos, Websites und Blogs finden. Genauer gesagt informieren sich $20,5 \%$ der Befragten über die neuesten High-End-Mode- oder Luxusartikeltrends aus den sozialen Medien (Deloitte, 2017). Fast alle Luxusmarken praktizieren Social Media Marketing, das definiert ist als "Marketingkommunikation über digitale Anwendungen, Plattformen und Medien, die Interaktion, Zusammenarbeit und das Teilen von Inhalten zwischen den Nutzern erleichtern" (Kim und Ko, 2012, S. 1480). Social Media bietet Luxusmarken neue Möglichkeiten, den Lead-Qualifizierungsprozess zu erleichtern, Markenbekanntheit zu schaffen und die Marke als "aufstrebende Marke" zu entwickeln. Laut Tennille Kopiasz, Senior Vice President of Marketing bei Parfums Christian Dior, "war der beste Weg für Luxusmarken, Verbraucher zu inspirieren, schon immer das Geschichtenerzählen. Da Instagram eine visuelle Storytelling-Plattform ist, passt der Link natürlich." (Instagram, 2017).

In diesem Rahmen untersucht diese Studie diesen unerforschten Bereich des Luxusmarkenmarketings auf Instagram und untersucht die wichtigsten Praktiken, die High-End-Marken derzeit in den sozialen Medien implementieren. Die spezifischen Ziele der Studie sind wie folgt: (1) das Konzept der Luxusmarken zu untersuchen, (2) die Prämissen des Social-Media-Marketings von Luxusmarken zu verstehen, die darauf 
abzielen, die Kundenbindung zu fördern, (3) die beliebten und spezifischen Marketingpraktiken von HighEnd-Marken auf Instagram zu bewerten, insbesondere in Bezug auf Influencer und Celebrity Endorsements.

\section{SCHLUSSFOLGERUNG}

\section{Theoretische Beiträge}

Dieses Papier befasste sich mit mehreren Zielen, die im Abschnitt Einführung hervorgehoben wurden, um die vorhandene Literatur in den folgenden Bereichen zu verbessern: (a) die Konzeptualisierung von "Luxusmarken", (b) Social-Media-Marketing von Luxusmarken, die auf die Kundenbindung abzielen, (c) spezifische Instagram-Marketingpraktiken im Kontext von Luxusmodemarken.

Grundlage für diese Studie war der Luxusmodesektor, in dem Social-Media-Strategien erfolgreich eingesetzt wurden, um Kunden und aufstrebende Konsumenten zu binden und zu erreichen. Akademische Arbeiten, die sich der Social-Media-Strategie von Mode-Luxusmarken widmen, sind begrenzt. Dieses Papier bietet neue Perspektiven auf dieses Thema. Frühere akademische Forschung (Godey et al., 2016; Kim und Ko, 2012; Phan et al., 2011) untersuchten, wie High-End-Marken an Instagram herankamen, um engere Beziehungen zu Kunden aufzubauen und die Markenbekanntheit im digitalen Umfeld zu erhöhen.

In diesem aktuellen Geschäftsumfeld sind Social-Media-Marketing-Aktionen Teil des Werbemixes im neuen Paradigma der Markenkommunikation (Mangold und Faulds, 2009). Während traditionelle Medien einen stärkeren Einfluss auf die Markenbekanntheit haben, beeinflusst die Social-Media-Kommunikation das Markenimage stark (Godey et al., 2016). Instagram ist aufgrund seiner visuellen Erweiterung, die es Marken ermöglicht, einen visuellen Storytelling-Ansatz zu verfolgen, auf Luxusmarken ausgerichtet. Instagram gilt mehr und mehr als das neue Ziel für Inspiration und eine neue Form des "Schaufensterbummels", da die Verbraucher dazu neigen, diese Social-Media-Plattform vor allem in der Entdeckungs- und Überlegungsphase zu konsultieren.

Social Media stellt eine wesentliche Ressource für Verbraucher in ihrem Entscheidungsprozess und für Vermarkter bei der Entwicklung und Pflege einer engen Marken-Kunden-Beziehung dar. In diesem Rahmen hat Instagram bei Verbrauchern und Vermarktern gleichermaßen an Popularität gewonnen. Instagram ist eine Social-Media-Plattform, die sich stark auf visuelles Storytelling konzentriert und das perfekte Medium für Produkte und Erlebnisse schafft. Luxusmarken haben diese Plattform auch eingeführt, um potenzielle Zielgruppen auf kreative Weise zu erreichen (Instagram, 2017).

\section{Auswirkungen auf das Management}

Diese Studie befasste sich mit mehreren Instagram-Marketingpraktiken, die im Kontext von Luxusmodemarken erfolgreich umgesetzt werden können. Durch seine Social-Media-Bemühungen kann ein Unternehmen die Wahrnehmungen, Einstellungen und Präferenzen der Verbraucher in Bezug auf seine Marke beeinflussen, indem es Praktiken verfolgt, die darauf abzielen, die Markenbekanntheit zu entwickeln und ein Markenimage zu schaffen, das bei den Zielkunden Ankommt. Godey et al. (2016) stellen fest, dass "Marken Social Media Marketing nicht mehr als eine Möglichkeit betrachten sollten, Verbraucher zu erreichen, sondern auch als ein wichtiges und kostengünstiges Image-Building-Tool".

Auf Instagram müssen Luxusmarken proaktiv originelle, aufmerksamkeitsstarke Inhalte entwickeln, die Kunden ansprechen. Darüber hinaus können Originelle Inhalte auch durch Partnerschaften mit Prominenten und Influencern erreicht werden. Die erstellten Inhalte zielen darauf ab, das Erbe der Marke zu verbessern und ihre Einzigartigkeit für potenzielle und aktuelle Kunden zu stärken sowie zu positiven Verhaltensweisen zu führen (Herrando et al., 2018). Tools wie Bumerangs, Highlights, Geschichten, Filter, Shopping usw. haben sich als interaktive Praktiken erwiesen, die Marken mehrere kreative Optionen bieten, um das Image der Luxusmarke, die Markenbekanntheit und das Engagement zu steigern.

Luxusmarken, die Instagram als Social-Media-Plattform angehen, die die Lücke zwischen dem Unternehmen und den gezielten Verbrauchern schließt, müssen sich auf das Storytelling konzentrieren. Auf Instagram können diese Storytelling-Funktionen durch den Feed, Videos, Geschichten und Live-Videos erreicht werden. Der Feed sollte redaktionell aussehen und zeitlos sein, verglichen mit Stories, wo momentane und kurzlebige Inhalte einen großen Wert haben. Zum Beispiel enthüllt Instagram in seinem 
Bericht von 2017, dass Burberry "die London Fashion Week mit 38\% der Diskussion dank seiner ansprechenden Inhalte hinter den Kulissen anführte".

Eine weitere nützliche Instagram-Praxis, die Luxusmodemarken in Betracht ziehen sollten, sind Hashtags. Hashtags stellen eine effektive Praxis dar, um die richtigen Zielgruppen auf Instagram zu mobilisieren. Darüber hinaus werden Hashtags für Forschung und Inspiration verwendet, von Vermarktern und Verbrauchern gleichermaßen. Nichtsdestotrotz spielt Celebrity Marketing eine wichtige Rolle bei der Kommunikation von Marketingbotschaften. Ebenso hat Influencer Marketing den Vorteil, High-EndMarken in einem informellen und zugänglichen Kontext zu präsentieren. Daher müssen Luxusmarken Instagram effektiv nutzen, um Zielgruppen zu erreichen.

\section{Grenzen und zukünftige Richtungen für den Ausbau der Forschung}

Eine wesentliche Einschränkung dieser Studie ist der Mangel an empirischen Beweisen für diese Aussagen. Zukünftige Forschung sollte sich auf die Entwicklung einer quantitativen Marktforschung mit Primärdaten konzentrieren, um den Grad der Instagram-Nutzung zu untersuchen, um Luxusmodekäufe im Allgemeinen und im Zusammenhang mit einer bestimmten High-End-Marke zu beeinflussen. Eine weitere Idee zur Erweiterung dieser Studie besteht darin, eine qualitative Forschung auf der Grundlage von TextMining und Stimmungsanalyse durchzuführen, um neue Informationen aus den Kommentaren der Nutzer über ihre Nutzung und Wahrnehmung der Präsenz von Luxusmodemarken auf Instagram zu sammeln. Die Studie könnte auch durch die Entwicklung einer interkulturellen Forschung erweitert werden, die die Nutzung von Instagram durch die Verbraucher untersucht und untersucht, wie sie Social-MediaMarketingpraktiken von Luxusmodemarken wahrnehmen.

\section{TRANSLATED VERSION: PORTUGUESE}

Below is a rough translation of the insights presented above. This was done to give a general understanding of the ideas presented in the paper. Please excuse any grammatical mistakes and do not hold the original authors responsible for these mistakes.

\section{VERSÃO TRADUZIDA: PORTUGUÊS}

Aqui está uma tradução aproximada das ideias acima apresentadas. Isto foi feito para dar uma compreensão geral das ideias apresentadas no documento. Por favor, desculpe todos os erros gramaticais e não responsacule os autores originais responsáveis por estes erros.

\section{INTRODUÇÃO}

À medida que a taxa de engajamento dos consumidores tende a diminuir, as marcas de luxo precisam reconsiderar sua estratégia de marketing digital e descobrir novas maneiras de maximizar seu retorno nos gastos de marketing. Uma estratégia de marketing de conteúdo precisa ser ajustada à plataforma social adequada para cada público-alvo, ao mesmo tempo em que atrai a atenção das principais demografias e é, e destaca chamadas de ação convincentes com um link integrado para o checkout.

As plataformas de mídia social representam um recurso essencial para os consumidores, seja para atividades de lazer, interagindo com amigos ou descobrindo novos produtos e serviços. Cada vez mais, as mídias sociais têm um papel importante na jornada do cliente ou no processo de tomada de decisão, influenciando todas as etapas desde a conscientização, consideração, avaliação, até a compra e recompra. Por essa razão, os profissionais de marketing estão altamente investidos no desenvolvimento de estratégias de mídia social voltadas para a aquisição, retenção e expansão de sua base de clientes. Para isso, a moda é considerada uma das indústrias mais importantes, uma vez que responde por uma parte significativa da economia mundial (McKinsey\&Co, 2019).

Nesta indústria, a moda de luxo desempenha um papel fundamental. Um relatório da Deloitte (2017) sobre o comportamento dos millennials sobre o comportamento do consumidor dos millennials encontrou 
millennials inclinados a comprar luxo dizem encontrar informações de fontes tradicionais, como revistas, até vídeos, sites e blogs. Mais especificamente, 20,5\% dos entrevistados descobrem as últimas tendências de moda high-end ou artigos de luxo das mídias sociais (Deloitte, 2017). Quase todas as marcas de luxo praticam o marketing de mídia social que é definido como "comunicação de marketing via aplicativos digitais, plataformas e mídias que facilitam a interação, colaboração e compartilhamento de conteúdo entre os usuários" (Kim e Ko, 2012, p. 1480). As mídias sociais oferecem novas oportunidades para marcas de luxo para facilitar o processo de qualificação de leads, criar consciência da marca e desenvolver a marca como uma "marca aspiracional". De acordo com Tennille Kopiasz, vice-presidente sênior de marketing da Parfums Christian Dior, "a melhor maneira de marcas de luxo inspirarem os consumidores sempre foi através da contação de histórias. Como o Instagram é uma plataforma de contação de histórias visuais, o link é um ajuste natural." (Instagram, 2017).

Neste quadro, este estudo examina essa área inexplorada de marketing de marcas de luxo no Instagram e examina as principais práticas que marcas high-end estão implementando atualmente nas mídias sociais. Os objetivos específicos do estudo são os seguintes: (1) examinar o conceito de marcas de luxo, (2) para entender as premissas de marketing de mídia social de marcas de luxo destinadas a impulsionar o engajamento do consumidor, (3) avaliar as práticas de marketing populares e específicas de marcas de ponta no Instagram, particularmente relacionadas a influenciadores e endosso de celebridades.

\section{CONCLUSÃO}

\section{Contribuições Teóricas}

Este artigo abordou múltiplos objetivos, destacados na seção Introdução, para aprimorar a literatura existente nas seguintes áreas: (a) a conceituação de "marcas de luxo", (b) marketing de mídia social de marcas de luxo voltadas para o engajamento do consumidor, (c) práticas específicas de marketing do Instagram no contexto de marcas de moda de luxo.

A base deste estudo foi o setor de moda de luxo, onde as estratégias de mídia social têm sido usadas com sucesso para engajar e alcançar clientes e consumidores aspiracionais. O trabalho acadêmico dedicado à estratégia de mídia social de marcas de luxo é limitado. Este artigo fornece novas perspectivas sobre este tema. Pesquisa acadêmica anterior (Godey et al., 2016; Kim e Ko, 2012; Phan et al., 2011) examinaram como marcas high-end abordaram o Instagram para desenvolver relações mais próximas com os clientes e aumentar a conscientização da marca em ambientes digitais.

Nesse ambiente de negócios atual, as ações de marketing de mídia social fazem parte do mix promocional no novo paradigma de comunicação da marca (Mangold e Faulds, 2009). Embora a mídia tradicional tenha um impacto mais forte na conscientização da marca, a comunicação nas mídias sociais influencia fortemente a imagem da marca (Godey et al., 2016). O Instagram é direcionado para marcas de luxo por causa de sua extensão visual que permite que as marcas tenham uma abordagem visual de contar histórias. Cada vez mais, o Instagram é considerado o novo destino da inspiração e uma nova forma de "compra de janelas", pois os consumidores tendem a consultar essa plataforma de mídia social especialmente na fase de descoberta e consideração.

As mídias sociais representam um recurso essencial para os consumidores em seu processo de tomada de decisão e para os profissionais de marketing no desenvolvimento e manutenção de um relacionamento próximo entre marca e cliente. Nesse quadro, o Instagram ganhou popularidade entre consumidores e profissionais de marketing. O Instagram é uma plataforma de mídia social altamente focada em contar histórias visuais que cria o meio perfeito para produtos e experiências. Marcas de luxo também adotaram essa plataforma para alcançar potenciais públicos de forma criativa (Instagram, 2017).

\section{Implicações Gerenciais}

Este estudo abordou múltiplas práticas de marketing do Instagram que podem ser implementadas com sucesso no contexto de marcas de moda de luxo. Por meio de seus esforços nas redes sociais, uma empresa pode influenciar as percepções, atitudes e preferências dos consumidores relacionados à sua marca, buscando práticas que visam desenvolver a conscientização da marca e criar uma imagem de marca que 
ressoe com clientes direcionados. Godey et al. (2016) afirmam que "as marcas não devem mais considerar o marketing de mídia social como uma maneira de alcançar os consumidores, mas também como uma importante e econômica ferramenta de construção de imagens".

No Instagram, marcas de luxo precisam ser proativas no desenvolvimento de conteúdo original e que atraia a atenção que envolva clientes. Além disso, o conteúdo original também pode ser alcançado através de parcerias com celebridades e influenciadores. O conteúdo criado tem como objetivo melhorar o legado da marca e reforçar sua singularidade para potenciais e clientes atuais, além de levar a comportamentos positivos (Herrando et al., 2018). Ferramentas como bumerangues, destaques, histórias, filtros, compras e assim por diante, provaram ser práticas interativas que dão às marcas múltiplas opções criativas para desenvolver a imagem da marca de luxo, a conscientização da marca e impulsionar o engajamento.

Marcas de luxo que abordam o Instagram como uma plataforma de mídia social que faz a ponte entre a empresa e os consumidores-alvo precisam focar a narrativa. No Instagram, essas capacidades de contar histórias podem ser alcançadas através do feed, vídeos, histórias e vídeos ao vivo. $\mathrm{O}$ feed deve ter um olhar editorial e ser atemporal, comparado ao Stories, onde o conteúdo momentâneo e efêmero tem um grande valor. Por exemplo, em seu relatório de 2017, o Instagram revela que a Burberry "liderou a Semana de Moda de Londres com 38\% da discussão graças ao seu conteúdo envolvente nos bastidores".

Outra prática útil do Instagram que as marcas de moda de luxo devem considerar são as hashtags. As hashtags representam uma prática eficaz para mobilizar o público certo no Instagram. Além disso, hashtags são usadas para pesquisa e inspiração, tanto por profissionais de marketing quanto por consumidores. No entanto, o marketing de celebridades tem um papel significativo na comunicação de mensagens de marketing. Da mesma forma, o marketing de influenciadores tem a vantagem de apresentar uma marca high-end em um contexto informal e acessível. Portanto, as marcas de luxo têm que usar o Instagram de forma eficaz para atingir o público-alvo.

\section{Limitações e Direções Futuras Para a Expansão da Pesquisa}

Uma das principais limitações deste estudo é a falta de evidências empíricas para essas proposições. Pesquisas futuras devem se concentrar no desenvolvimento de uma pesquisa de marketing quantitativo com dados primários para examinar o nível de uso do Instagram para influenciar compras de moda de luxo, em geral e relacionadas a uma determinada marca high-end. Outra ideia para ampliar este estudo é realizar uma pesquisa qualitativa baseada na mineração de texto e análise de sentimentos para coletar novas informações dos comentários dos usuários sobre seu uso e percepção da presença de marcas de moda de luxo no Instagram. $\mathrm{O}$ estudo também pode ser estendido no desenvolvimento de uma pesquisa transcultural que examine o uso do Instagram pelos consumidores e como eles percebem as práticas de marketing de mídia social de marcas de moda de luxo. 